\title{
Susceptibility to paratuberculosis infection in cattle is associated with single nucleotide polymorphisms in Toll-like receptor 2 which modulate immune responses against Mycobacterium avium subspecies paratuberculosis
}

\author{
A. Koets ${ }^{\mathrm{a}, \mathrm{c}, *}$, W. Santema ${ }^{\mathrm{a}, \mathrm{c}}, \mathrm{H}_{\text {. Mertens }}{ }^{\mathrm{a}}$, D. Oostenrijk ${ }^{\mathrm{a}, 1}$, M. Keestra $^{\mathrm{b}, 2}$, M. Overdijk $^{\mathrm{a}, 3}$, \\ R. Labouriau ${ }^{\mathrm{d}}$, P. Franken ${ }^{\mathrm{e}}$, A. Frijters ${ }^{\mathrm{f}}$, M. Nielen ${ }^{\mathrm{c}}$, V. Rutten ${ }^{\mathrm{a}, \mathrm{g}}$ \\ ${ }^{a}$ Department of Infectious Diseases and Immunology, Immunology Division, P.O. Box 80.165, 3508TD Utrecht, The Netherlands \\ ${ }^{\mathrm{b}}$ Department of Infectious Diseases and Immunology, Infectionbiology Division, P.O. Box 80.165, 3508TD Utrecht, The Netherlands \\ ${ }^{\mathrm{c}}$ Department of Farm Animal Health, Epidemiology Division, Faculty of Veterinary Medicine, Utrecht University, P.O. Box 80.151, 3508TD Utrecht, The Netherlands \\ ${ }^{\mathrm{d}}$ Research Group for Bioinformatics Genetics and Statistics, Department of Genetics and Biotechnology, Faculty of Agricultural Sciences, University of Aarhus, P.O. \\ Box 50, DK-8830 Tjele, Denmark \\ ${ }^{\mathrm{e}}$ GD Animal Health Service, P.O. Box 9, 7800 AA Deventer, The Netherlands \\ ${ }^{\mathrm{f}}$ CRV Research \& Development, P.O. Box 454, 6800 AL Arnhem, The Netherlands \\ ${ }^{\mathrm{g}}$ Department of Veterinary Tropical Diseases, Faculty of Veterinary Science, University of Pretoria, Private Bag X04, Onderstepoort 0110, South Africa
}

\section{A R T I C L E I N F O}

Keywords:

Toll-like receptor 2

Johne's disease

Paratuberculosis

Innate immunity

Genetic susceptibility

\begin{abstract}
A B S T R A C T
Paratuberculosis is a chronic intestinal infection in ruminants, caused by Mycobacterium avium subspecies paratuberculosis (Map). To study the role of host genetics in disease susceptibility, the Toll-like receptor 2 (TLR2) gene, selected based on its potential role in immunity to mycobacterial infections, was analyzed for single nucleotide polymorphisms (SNP) and their potential association with disease.

For SNP discovery and to study SNP association with disease, a case-control study including 24 cows from farms with paratuberculosis was conducted. Sequence analysis of the TLR2 genes from 12 paratuberculosis-infected animals and 12 age-matched healthy herd mates revealed 21 different SNP. The TLR2-1903 T/C SNP was significantly associated with resistance to Map. This and four additional TLR2 SNP were studied in a subsequent observational field study with 553 cows from farms with paratuberculosis. The allelic distribution of the TLR2-1903 T/C SNP was confirmed to be significantly different between the infected and non-infected animals. For the TLR2-1903 T/C SNP the odds ratio was calculated, and similar to the dominance model in the association study, the CT and CC genotypes were compared to the TT genotype. Cows with the TLR2-1903 T/C mutation (i.e., the CT and CC genotypes) were at 1.7 (95\% CI: 1.2, 2.8) times the odds of being Map-infected compared to cows with the TT genotype. In in vitro functional assays, monocyte-derived macrophages from animals with a TLR21903 TT genotype produced more IL12p40 and IL1 $\beta$ when stimulated with Map compared to cells derived from TLR2-1903 CT and CC genotypes. Also, T cell proliferative responses to mycobacterial antigens were higher in animals with a TLR21903 TT genotype.
\end{abstract}

\footnotetext{
* Corresponding author.

E-mail address: a.p.koets@uu.nl (A. Koets).

1 Present address: Department of Pathology, UMCU, P.O. Box 85500, 3508 GA Utrecht, The Netherlands.

2 Present address: Department of Medical Microbiology and Immunology, University of California, One Shields Avenue, Davis, CA 95616, USA.

3 Present address: Genmab B.V., P.O. Box 85199, 3508 AD Utrecht, The Netherlands.
} 
In conclusion, we have found a significant association between SNP TLR2-1903 T/C in the bovine TLR2 gene and bovine paratuberculosis infection. This SNP and other genetic markers could be useful in marker-assisted breeding strategies as an additional tool in paratuberculosis control strategies. In addition, the functional studies suggest that genetic polymorphisms in bovine TLR2 which result in higher macrophage activity may contribute to enhanced $\mathrm{T}$ cell activation and a lower susceptibility to paratuberculosis in cattle.

(c) 2009 Elsevier B.V. All rights reserved.

\section{Introduction}

Paratuberculosis (Johne's disease) is a chronic progressive infection of the intestinal mucosa in ruminants. It is caused by Mycobacterium avium subspecies paratuberculosis (Map), an intracellular pathogen residing in host macrophages. The bacteria are excreted in feces and milk of infected animals in later stages of the disease. The disease constitutes a worldwide threat to dairy cattle health. In addition, the presence of Map in milk is a potential public health risk as Map has been implicated in the etiology of human Crohn's disease (Greenstein, 2003). In humans, host genetic variation has been known to influence resistance to infection and substantially influence the phenotype of infection following exposure to mycobacteria (Casanova and Abel, 2002). While in mice and humans several genes involved in resistance to mycobacterial infections have been studied in detail, the data regarding cattle are scarce. Koets et al. (2000) indicated the presence of genetic variation in the susceptibility to paratuberculosis. Recent studies point to various chromosome regions or quantitative trait loci (QTL) associated with paratuberculosis (Gonda et al., 2006, 2007). Polymorphisms in two genes associated with innate immune responses, Solute Carrier family 11 member 1 (SLC11A1) (Estonba et al., 2005) and Caspase recruitment domain 15 (CARD15) (Pinedo et al., 2008), have been described as potentially involved in the mechanism of resistance to Map infection in cattle.

Infection of young calves mostly occurs after oral uptake of Map from food or the environment. After Map has crossed the intestinal barrier by $M$ cell uptake, it is recognized by phagocytes, such as macrophages and dendritic cells (DC), as part of the innate immune response against pathogens (Coussens, 2004). Toll-like receptors (TLR) and cytosolic Nucleotide Oligomerization Domain (NOD)-like receptors are, among other pattern recognition receptors, involved in the recognition of mycobacteria, as well as in stimulating phagocytosis and the development of cell-mediated immunity (Quesniaux et al., 2004). We hypothesize that inefficient recognition of Map and failure in the subsequent innate and adaptive immune responses can be caused by defects in genes coding for TLR. TLR2 has been described as one of the main receptors for mycobacteria, and in humans, mutations in TLR2 have been detected and associated with higher susceptibility to tuberculosis (Lorenz et al., 2000; Kang and Chae, 2001; Ogus et al., 2004). After uptake, Map is able to evade the destructive processes by inhibition of phagolysosome fusion and is able to replicate inside the macrophages
(Kuehnel et al., 2001; Hostetter et al., 2003; Weiss et al., 2004) and possibly also inside DC. These antigen-presenting cells play a crucial role in the induction of adaptive immune responses and the polarization of $\mathrm{T}$ helper cells (Kalinski et al., 1999).

Resistance to intracellular pathogens such as Map predominantly depends on a T helper 1 (Th1) induction and effector phase of the adaptive immune system. Production of interferon gamma (IFN $\gamma$ ) by natural killer (NK) and T cells is one of the first detectable signs of infection, indicating activation of cell-mediated immunity (CMI). During the first stages of paratuberculosis (latent phase), a high level of CMI is present. Some cows remain in this stage for years; others rapidly progress to the subclinical and clinical stages of disease (Alzuherri et al., 1997). In these later phases, the Th1 CMI declines to an undetectable low level and the amount of apparently non-protective antibodies increases. The loss of protective CMI leads to a widely disseminated infection and uncontrolled growth of mycobacteria in macrophages, causing the clinical manifestation of paratuberculosis (Koets et al., 2002). Hence, following a prolonged period of chronic diarrhoea, weight loss and decreased milk production, cows become emaciated and may die from this protein-loosing enteropathy (Coussens, 2004). Regulatory T cells may also play a role in suppression of Th1 activity, amongst others via the production of the immunosuppressive IL-10 (Weiss et al., 2005), and TLR2 may play a role in this process (Weiss and Souza, 2008). In cattle, TLR2 has been found to be expressed by myelomonocytic cells (monocytes, DC, macrophages and polymorphonuclear cells) and it is undetectable in resting or activated lymphoid subsets (Werling et al., 2004, 2006). Therefore, TLR2 may play a role in the recognition of intestinal pathogens, like mycobacteria, when these pathogens have crossed the intestinal epithelial barrier. Expression of TLR2 on bovine macrophages is eight times higher than that on DC (Werling et al., 2004). As bovine macrophages are the primary target cells in which Map is able to survive and replicate, the TLR2 function may be critically important in paratuberculosis (Weiss et al., 2007).

The aim of the present study was to identify single nucleotide polymorphisms (SNP) in TLR2 genes and to associate these SNP with different phenotypes of bovine paratuberculosis, as an indicator for high and low susceptibility to disease. Existence of genetic variants with different resistance to paratuberculosis may enable marker-assisted selection for Map resistance. The functional consequences of these mutations may shed light on the pathogenic mechanisms involved in bovine paratuberculosis. 


\section{Materials and methods}

\subsection{Study design and sources of bovine DNA}

The DNA used in the case-control study was derived from 24 Holstein Friesian cows, which originated from 12 different Dutch farms. The study population was divided into cases and controls; 12 cows in each group. Data from routine semi-quantitative fecal culture diagnostic tests (Jorgensen, 1982), conducted by the Dutch Animal Health Service, were used for primary classification. The cases shed Map in their feces and showed clinical signs of paratuberculosis, which were confirmed by histopathology. The control cows were healthy age-matched herd mates of the cases which did not shed Map in their feces and were serologically negative for Map. Although the control cows were kept in the same environment and were exposed to Map similar to the cases, they did not get infected, or cleared the infection. The cows with clinical signs were defined as cows with a high susceptibility to paratuberculosis; the healthy control herd mates as having a low susceptibility.

For confirmation studies following the SNP discovery in the case-control study, blood samples were collected from 553 adult cattle originating from eight other farms with endemic paratuberculosis. These farms participated in a national program and their paratuberculosis status had been monitored for the last four years. Data from routine diagnostic tests for paratuberculosis conducted by the Dutch Animal Health Service, i.e., semi-quantitative fecal culture and absorbed ELISA (Institute Pourquier, Montpellier, France), were used to group animals by infection status. Data from five to seven consecutive samplings over the period between January 2001 and November 2004 were analyzed. Animals were only considered negative (uninfected) when all tests at all time points were negative. All other animals were considered to be infected with Map. Due to the nature of the Dutch Animal Health Service survey, no data were recorded regarding the clinical signs of paratuberculosis in these animals.

In 2007 an additional 50 animals were identified as being infected with Map on 27 different farms (other than the farms used for previous studies) using a commercially available diagnostic ELISA (Institute Pourquier, Montpellier, France) and confirmed by semi-quantitative fecal culture by the Dutch Animal Health Service as described above. These animals were genotyped, and lymphocytes isolated from diagnostic samples taken from these animals were used in immune function studies conducted as part of this investigation.

\subsection{Preparation of genomic DNA}

Peripheral blood mononuclear cells (PBMC, from 22 cows) and whole blood (from two cows in the case-control study and 553 cows in the confirmation study) were used as a source of genomic DNA. The PMBC had been obtained from whole blood by density gradient centrifugation as published previously (Koets et al., 2002). The DNA from purified PBMC or whole blood was isolated using the DNAWizard-Kit (Promega, Madison, WI, USA) according to manufacturer's instructions and spectrophotometrically tested for yield and purity.

\subsection{TLR2 primer design and DNA amplification}

For SNP discovery in the case-control study primers were designed using Primer 3 web-based software (Rozen and Skaletsky, 2000) on the published sequence of TLR2 (AF368419). The coding (mRNA) sequence of TLR2 [2354 nuleotides (nt)] is located in one exon (exon 2) of the TLR2 gene which is located on chromosome Bta17. Five sets of primers were designed to generate amplicons by PCR between 526 and 822 nt in size from genomic DNA and covering the exon 2 completely. The primers used in this study are listed in Table 1. PCR reactions were performed in a total volume of $40 \mu \mathrm{L}$ containing $100 \mathrm{ng}$ of genomic DNA, 1.0 U pfu-polymerase (Promega, Madison, WI, USA), $10 \times$ reaction buffer with $\mathrm{MgSO}_{4}$ (Promega, Madison, WI, USA), and final concentrations of $200 \mu \mathrm{M}$ dNTPs (Invitrogen, Carlsbad, CA, USA) and $0.2-0.8 \mu \mathrm{M}$ of forward and reverse primer (Invitrogen, Carlsbad, CA, USA). The PCR reaction mix was subjected to 30 cycles of amplification in an iCycler PCR system (Bio-Rad, Hercules, CA, USA). The cycling profile started with a 2 -min step at $95^{\circ} \mathrm{C}$, followed by 30 cycles of $30 \mathrm{~s}$ at $95^{\circ} \mathrm{C}$ denaturation, $30 \mathrm{~s}$ at $41-56^{\circ} \mathrm{C}$ annealing (primer dependant) and $30 \mathrm{~s}$ at $72{ }^{\circ} \mathrm{C}$ extension. The PCR products were analyzed by electrophoresis in $1 \%$ agarose gels, stained with ethidium bromide, and expected size was checked using appropriate molecular weight DNA standards (Massruler, Fermentas, Burlington, Ontario, Canada).

Table 1

Primer sequences of the primers used to amplify five overlapping parts of bovine Toll-like receptor 2 (TLR2) spanning the coding sequence of TLR2 in exon 2.

\begin{tabular}{llc}
\hline Primer & Sequence & Start position \\
\hline TLR2-1F & GGA CAA TGC CAC GTG CTT & 192 \\
TLR2-1R & GCA CTG ATC TCA AGC TCC TCA AG & 744 \\
TLR2-2F & TGA GGA GCT TGA GAT CAG TG & 724 \\
TLR2-2R & ACT GTG TAT CCT TGT GCT GG & 1546 \\
TLR2-3F & CCT AGG TAA TGT GGA GAC G & 111 \\
TLR2-3R & AAG GAG GCA TCT GGT AGA G & $\mathbf{1 1}$ \\
TLR2-4F & CCA GCA CAA GGA TAC ACA GT & 1686 \\
TLR2-4R & CTT CAT GTA CCA CAG TCC GT & 1527 \\
TLR2-5F & TTC CTG TTG CTC CTG CTC AC & 2053 \\
TLR2-5R & GAC CAC CAC CAG ACC AAG AC & 1991 \\
\hline
\end{tabular}

$\mathrm{bp}=$ basepair. 


\subsection{SNP discovery}

PCR products were bidirectionally sequenced by VIB Genetic Service Facility, Antwerp, Belgium, using ExoSAPIT (USB, Cleveland, OH, USA) for PCR cleanup and BigDye Terminator v3.1 Cycle sequencing reaction on an automated DNA Analyzer (Applied Biosystems, Foster City, CA, USA). Primers used for sequencing were the same as used for DNA amplification (Table 1). The reference sequence was chosen based on the available Genbank bovine TLR2 sequence AF368419. The sequences of the PCR products were compared with these reference sequences by novoSNP software, which allowed detection of sequence variations like SNP (Weckx et al., 2005).

\subsection{SNP detection}

Non-monomorphic TLR2 variants with a frequency greater than $10 \%$ were selected for analysis in the larger cattle population $(n=553)$. The SNP genotyping was again outsourced to the VIB Genetic Service Facility, Antwerp, Belgium, and performed using a mass-spectrometry based SNP genotyping technique (Mass-array SNP genotyping, Sequenom, San Diego, CA, USA).

\subsection{Measurement of bovine TLR2 induced NFKB activity}

To study the functional consequences of different haplotypes of TLR2 on intracellular signaling following ligand recognition, a Nuclear Factor $\kappa \mathrm{B}(\mathrm{NF} \kappa \mathrm{B})$-driven luciferase reporter assay was used as previously published (Keestra et al., 2007).

In short, primers were designed on the coding region of bovine TLR2 (GenBank accession number AF368419), downstream the region coding for the signal peptide (first 60 bases of the coding sequence). The forward and reverse primers were TLR2F 5'-GAATTCGGCTGTCATCATCCTGCTCA$3^{\prime}$, containing an enzyme-restriction site (EcoRI), and TLR2R 5'-GACCACCACCAGACCAAGAC-3', respectively. The amplified TLR2 DNA was ligated into a pGEMTeasy-vector (Promega, Madison, WI, USA) and E. coli DH5 $\alpha$-cells (Invitrogen, Carlsbad, CA, USA) were transformed with this vector. The presence of TLR2 in positive clones was confirmed by PCR. The TLR2-containing plasmid was isolated from confirmed $\mathrm{DH} 5 \alpha$-clones, and digested using the enzymes EcoRI and BamHI. This fragment was isolated from gel using Zymoclean Gel DNA Recovery Kit (Zymo Research, Orange, CA, USA) and ligated in the p-FLAG-CMV1 expression-vector (Sigma-Aldrich, St. Louis, MO, USA). Insert identity of the cloned genes in the pFLAG-CMV-1 vector was confirmed by sequencing.

The human embryonic kidney cell line 293 (HEK293 cells) was obtained from the American Type Culture Collection, USA, and grown according to instructions provided. HEK293 cells were plated at $3.8 \times 10^{5}$ cells/well in a 24-wells plate (Costar, Cambrdige, MA, USA) on the day before transfection. The cells were transfected using Lipofectamine 2000 (Invitrogen, Carlsbad, CA, USA) with $0.1 \mu \mathrm{g}$ NFкB-Luciferase reporter construct (Stratagene, La Jolla, CA, USA), $0.14 \mu \mathrm{g}$ PDM-LacZ plasmid and $0.26 \mu \mathrm{g}$ of the p-FLAG-CMV-1-vector, containing the relevant TLR2
DNA insert. For mock-transfection, the HEK293 cells were transfected with the $0.1 \mu \mathrm{g}$ NFкB-Luciferase reporter construct and $0.14 \mu \mathrm{g} \mathrm{LacZ}$ as well and $0.26 \mu \mathrm{g}$ of the pFLAG-CMV-1-vector, without the TLR2 DNA insert.

Forty-eight hours after transfection, the cells were stimulated with various concentrations of synthetic tripalmitoylated lipopeptide PAM3CSK4 (palmitoyl-3-cysteine-serine-lysine-4, 200 and $500 \mathrm{ng} / \mathrm{ml}$ ), PAM2CSK4 (200 and $500 \mathrm{ng} / \mathrm{ml}), \operatorname{Map}\left(0.7 \times 10^{6}, 1.4 \times 10^{6}\right.$ and $2.8 \times 10^{6}$ bacteria/ml, multiplicity of infection ( $\mathrm{moi}$ ) of two, four and eight, respectively) and recombinant Map Hsp70 (1, 5 and $10 \mu \mathrm{g} / \mathrm{ml}$ ) in $500 \mu \mathrm{l}$ medium for $5 \mathrm{~h}$. The cells were then lysed and luciferase activity was measured, using the luciferase assay system (Promega, Madison, WI, Madison, USA) according to the manufacturer's instructions, in relative light units (RLU) in a luminometer (Turner Design, Sunnyvale CA, USA). Luciferase activity was corrected via co-transfected LacZ-activity to account for differences in transfection efficiency. LacZ-activity was measured spectrophotometrically (Ultrospec 2000 UV/vis spectrophotometer, Amersham Pharmacia Biotech Ltd, Buckinghamshire, England). Relative luciferase activity was defined as the ratio of the luciferase activity measured in cells after stimulation and cells without stimulation.

\subsection{Flowcytometric evaluation of TLR2 expression in HEK293 cells}

TLR2 protein expression levels, prior to and after stimulation of the TLR2 protein with the ligands described above, were determined on single cell level by flowcytometry. In short, transfected HEK293 cells and mocktransfected controls were stained with the M2 antibody which recognizes the N-terminal expressed FLAG epitope, present on the N-terminus of the extracellular LRR of expressed TLR2 molecules, according to manufacturer's instructions (Sigma-Aldrich, St. Louis, MO, USA). Alexa633 labeled goat-anti-mouse antibodies were used for indirect staining according to manufacturer's instructions (Molecular Probes - Invitrogen, Carlsbad, CA, USA). Fluorescence of 20,000 cells per treatment was acquired on a FacsCalibur (BD Biosciences, San Jose, CA, USA) and analyzed using Cellquest software (BD Biosciences, San Jose, CA, USA).

\subsection{Quantitative RT-PCR gene expression analysis of bovine monocytes}

Heparinized blood was collected from 10 cows with either a resistant $(n=5)$ or a susceptible $(n=5)$ TLR2 genotype, defined as, respectively, the absence or presence of the SNP01 TLR2 $1903 \mathrm{~T} / \mathrm{C}$. Bovine monocytes were isolated using an anti-CD14 antibody-coated magnetic bead positive selection system (MACS, Miltenyi Biotech), grown overnight in a humidified incubator, stimulated with Map (moi 10:1), MAA (moi 10:1) or left unstimulated and harvested for gene expression analysis after $6 \mathrm{~h}$ incubation as described in detail previously with minor modifications (Langelaar et al., 2005). Total RNA was isolated using the RNEasy kit and DNAse treatment was applied to all samples according to manufacturer's 
Table 2

Single nucleotide polymorphisms (SNP) detected in bovine Toll-like receptor 2 (TLR2) gene sequences derived from 24 cattle in a case-control study (cases were cows with clinical signs of paratuberculosis and controls healthy age-matched herd mates).

\begin{tabular}{|c|c|c|c|c|c|c|}
\hline \multirow[t]{2}{*}{ SNP position ${ }^{\mathrm{a}}$} & \multirow[t]{2}{*}{ Codon change } & \multirow[t]{2}{*}{ Amino acid change } & \multicolumn{2}{|c|}{ SNP frequency } & \multirow[t]{2}{*}{ Fisher's exact $P$-value ${ }^{\mathrm{b}}$} & \multirow[t]{2}{*}{ TLR2 domain } \\
\hline & & & Case & Control & & \\
\hline 382 & aat $\rightarrow$ aac & $\mathrm{N}=\mathrm{N}$ & 0 & 1 & n.s. & putative LRR \\
\hline 385 & gat $\rightarrow$ gag & $D \rightarrow E$ & 5 & 8 & n.s. & putative LRR \\
\hline 398 & $g g c \rightarrow a g c$ & $G \rightarrow S$ & 0 & 1 & n.s. & putative LRR \\
\hline 651 & $\operatorname{cgg} \rightarrow \operatorname{cag}$ & $R \rightarrow Q$ & 4 & 7 & n.s. & putative LRR \\
\hline 798 & $a g c \rightarrow a a c$ & $S \rightarrow N$ & 4 & 7 & n.s. & putative LRR \\
\hline 827 & $a t t \rightarrow g t t$ & $I \rightarrow V$ & 7 & 8 & n.s. & putative LRR \\
\hline 1141 & $\operatorname{cgg} \rightarrow \operatorname{cgt}$ & $\mathrm{R}=\mathrm{R}$ & 0 & 1 & n.s. & putative LRR \\
\hline 1174 & $\mathrm{cat} \rightarrow \mathrm{caa}$ & $H \rightarrow Q$ & 0 & 1 & n.s. & putative LRR \\
\hline 1206 & $a g a \rightarrow a a a$ & $R \rightarrow K$ & 0 & 1 & n.s. & putative LRR \\
\hline 1446 & $a a c \rightarrow a g c$ & $N \rightarrow S$ & 0 & 1 & n.s. & putative LRR \\
\hline 1504 & gga $\rightarrow$ ggc & $G=G$ & 0 & 1 & n.s. & putative LRR \\
\hline 1828 & $\mathrm{ttc} \rightarrow \mathrm{ttt}$ & $\mathrm{F}=\mathrm{F}$ & 2 & 6 & 0.097 & putative LRR \\
\hline 1884 & $\operatorname{cgc} \rightarrow \mathrm{cac}$ & $R \rightarrow H$ & 0 & 1 & n.s. & putative LRR \\
\hline 1903 & cat $\rightarrow$ cac & $\mathrm{H}=\mathrm{H}$ & 2 & 7 & 0.045 & putative LRR \\
\hline 1975 & $\mathrm{gct} \rightarrow \mathrm{gcc}$ & $A=A$ & 0 & 1 & n.s. & transmembrane \\
\hline 1978 & $\mathrm{gcg} \rightarrow \mathrm{gct}$ & $A=A$ & 0 & 1 & n.s. & transmembrane \\
\hline 2191 & $\mathrm{cac} \rightarrow \mathrm{cag}$ & $H \rightarrow Q$ & 0 & 1 & n.s. & TIR \\
\hline 2221 & cat $\rightarrow$ cac & $\mathrm{H}=\mathrm{H}$ & 0 & 1 & n.s. & TIR \\
\hline 2251 & att $\rightarrow$ atc & $\mathrm{I}=\mathrm{I}$ & 0 & 1 & n.s. & TIR \\
\hline 2410 & gag $\rightarrow$ gaa & $E=E$ & 0 & 1 & n.s. & TIR \\
\hline 2491 & $\mathrm{ccc} \rightarrow \mathrm{cct}$ & $\mathrm{P}=\mathrm{P}$ & 0 & 1 & n.s. & TIR \\
\hline
\end{tabular}

The location of SNP in the molecule, the consequence of the SNP in the codon, the predicted amino acid change, the frequency in cases and controls, and the functional domain affected are indicated.

a All SNP identified in bovine TLR2 based on sequencing TLR2 exon 2 from 24 animals; non-synonymous SNP are in italics.

b The level of statistical significance was set at $P<0.05$; n.s. = not significant; LRR = Leucine Rich Repeats, TIR = Toll/interleukin- 1 receptor (TIR) domain.

instructions (Qiagen). Total RNA quality and quantity was measured spectrophotometrically (Nanodrop 1000 system). RNA was transcribed to cDNA using the iScript kit according to instructions provided (Biorad Laboratories). Quantitative PCR was done on an IQ5 thermocycler (Biorad Laboratories) using a using iTaq SYBR Green Supermix with ROX (Biorad Laboratories). Transcription of IL1 $\beta$, IL6, TNF $\alpha$, IL4, IL10, IFN $\gamma$, IL12p40, TLR2, TLR9 and $\beta 2-$ microglobulin was examined. All PCR reactions were set up in 96-well microAmp plates (Applied Biosystems, CA, USA) using predetermined forward and reverse primer concentrations as indicated in Table 2 in a reaction volume of $25 \mu \mathrm{l}$. After $10 \mathrm{~min}$ ' dissociation at $95^{\circ} \mathrm{C}$, the reactions were cycled 40 times at $95^{\circ} \mathrm{C}$ for $15 \mathrm{~s}$ and $60{ }^{\circ} \mathrm{C}$ for $1 \mathrm{~min}$. Melting point analysis was done after the last cycle to verify the amplification specificity by cooling samples to $60{ }^{\circ} \mathrm{C}$ and then increasing the temperature to $95^{\circ} \mathrm{C}$ at $1{ }^{\circ} \mathrm{C} /$ min. A single product at a specific melting temperature was found for each target gene.

The relative transcription ratio of each target gene was calculated based on the PCR efficiency and the CT deviation of transcription in resistant genotypes versus a control (sensitive genotype) and normalized to $\beta 2$-microglobulin transcription as reference gene for each antigenic stimulation, using the quantification software REST $\odot$ (Relative Expression Software Tool) according to published methods (Pfaffl et al., 2002).

\subsection{Lymphocyte proliferation assay}

Heparinized blood was collected from 40 cows with either a resistant $(n=25)$ or a susceptible $(n=15)$ TLR2 genotype, defined as, respectively, the absence or presence of the SNP01 TLR2 1903 T/C. These cows were clinically healthy animals, infected with Map, as confirmed by routine fecal culture. The average age of the cattle was 4.9 years and did not differ significantly between the two groups. PBMC were isolated using density gradient centrifugation and used in lymphocyte stimulation assays according to methods published previously (Koets et al., 1999). In short, cell suspensions ( $\left.100 \mu \mathrm{l} 2 \times 10^{6} \mathrm{PBMC} / \mathrm{ml}\right)$ were cultured with $100 \mu \mathrm{l}$ antigen (avian PPD, $10 \mu \mathrm{g} / \mathrm{ml}$ ), medium negative controls as well as concanavalin $\mathrm{A}$ $(2.5 \mu \mathrm{g} / \mathrm{ml})$ control in triplicates in a 96-well microtiter plate (Corning Costar Corp., Acton, MA, USA) at $37^{\circ} \mathrm{C}$ and $5 \%$ $\mathrm{CO}_{2}$ in a humidified incubator for four days. Finally, $0.4 \mu \mathrm{Ci}$ ${ }^{3} \mathrm{H}$-thymidine (Amersham, Buckinghamshire, UK) was added to each well and cells were cultured for an additional $18 \mathrm{~h}$. Subsequently, incorporation of ${ }^{3} \mathrm{H}$-thymidine was measured by micro-Betaplate liquid scintillation counting (EG\&G Wallac, Turku, Finland), and expressed as counts per minute (cpm). Stimulation indices were calculated by dividing cpm of stimulated wells by cpm of the medium control wells for each animal.

\subsection{Statistical analyses}

Statistical analyses were done using the $\mathrm{R}$ software package ( $R$ Development Core Team, 2008), with the exception of post hoc power calculations for the casecontrol and observational field studies which were performed using WinEpiscope 2.0.

To determine whether an association between genotype and susceptibility to paratuberculosis could be identified, we investigated the allelic association of SNP in the case-control group. Fisher exact tests with correc- 
tion for multiple comparisons were used to compare the SNP-distribution in both groups. A $P$-value of $<0.05$ was considered significant.

The observational field study data were analyzed using binomial logistic models with the response variable given by a binary indicator of the paratuberculosis diagnosis. Several combinations of the five informative SNP markers were used as discrete explanatory variables, as described below. The SNP markers entered in the model as dominant markers, that is, each factor had two levels: homozygous of wild type or a genotype containing at least one variation allele (i.e., heterozygous or homozygous of variation type). All the logistic regressions contained a normal random factor representing the farm.

A global test of association was performed by comparing, via the likelihood ratio test, a full model containing simultaneously factors representing each of the five informative SNP markers (and a factor representing the farm) against a null model containing only a factor representing the farm. Interactions between farm and the SNP markers were tested for.

Following the methodology described in Labouriau et al. (2008) a series of logistic models containing combinations of the five informative SNP markers was fit in order to identify possible associations with complex haplotypes. The model with smaller Bayesian Information Criterion (BIC) or Schartz Information Criterion was chosen among all the possible models formed with combinations of the five SNP markers (Haughton, 1988). Similar results were obtained when using the Akaike Information Criterion (Burnham and Anderson, 2002).

Functional immune response differences between animals with the two different TLR2 haplotypes in the luciferase reporter assay, lymphocyte stimulation assay and antibody assays were analyzed using a Students T-test. A $P$-value of $<0.05$ was considered significant.

\section{Results}

\subsection{Identification of disease associated SNP in the case- control population}

In total, five PCR amplicons from exon 2 of the TLR2 gene were sequenced from each of the 24 animals in the case-control study. Using the novo-SNP program, 82 polymorphisms were identified in 21 unique positions in the coding sequence (exon 2) of bovine TLR2 from 12 clinical (cases) and 12 non-clinical (controls) cows. The results are summarized in Table 2 . The 21 different SNP were detected in five amplicons; each amplicon contained four $(n=4)$ or five $(n=1)$ SNP. As 15 of the detected SNP were only found in one of 24 animals these were considered non-informative for the present study. Except for one SNP $(827 \mathrm{~A} / \mathrm{G})$, the animals were heterozygous for the SNP (97.6\% heterozygosity). The majority of these SNP were transitions [64 of 82 SNP (78\%), largely determined by $\mathrm{G}>\mathrm{A}$ and $\mathrm{T}>\mathrm{C}$ substitutions]. Fifteen $\mathrm{T} \leftrightarrow \mathrm{G}$ transversions were identified (18.3\%). In this study, the ratio transitions: transversions was 78.0:18.3 (4:1). Fifty-six of the 82 SNP (68.3\%) were non-synonymous and cause an amino acid substitution (Table 2 ). The ratio non-synony- mous:synonymous was 56:26 (2.2:1). Eighty-two SNP give an average SNP frequency of 3.4 SNP per TLR2 gene sequenced (1 SNP per $689 \mathrm{bp}$ ).

Seventy-five SNP (91\%) were located in the putative LRR domain, which is used for ligand recognition. In one nonclinical animal two SNP were detected in the transmembrane domain and five in the TIR domain, the intracellular signalling domain. The average SNP frequency per domain indicated that the LRR domain had one SNP per $545 \mathrm{bp}$, the transmembrane domain one SNP per $756 \mathrm{bp}$ and the TIR domain one per 2098 bp. Fifty-six non-synonymous SNP were found ( 10 different ones); 55 of 56 were located in the putative LRR domain, none in the transmembrane domain and one in the TIR domain. The average frequency of nonsynonymous SNP per domain was one SNP per $730 \mathrm{bp}$ in the LRR domain and one SNP per $10488 \mathrm{bp}$ in the TIR domain.

In summary, the SNP at basepair positions 385,651 , 798,1828 , and 1903 were seen frequently in the casecontrol study. Based on the Fisher's exact tests the TLR21903 T/C SNP showed significantly different allelic distribution $(P=0.045)$ between cases and controls, and an OR of six. In addition, a marginally statistically significant association was found for the TLR2-1828 C/T SNP $(P=0.097)$ (Table 2).

\subsection{Confirmation of SNP disease associations in the observational field study}

The five SNP potentially associated with susceptibility to paratuberculosis were studied in 553 cattle originating from eight farms with endemic paratuberculosis. Analysis of age distribution between paratuberculosis negative ( $n=308$, average age $5.5 \pm 1.8$ years) and positive cattle ( $n=245$ average age $5.2 \pm 1.8$ years at final sampling) showed no significant differences. The average prevalence of paratuberculosis positive animals at the farm level at the last time of sampling was $32.2 \%$ and ranged from $22.2 \%$ to $56.0 \%$. On these eight farms the prevalence of infection was increasing in the 4 years of observations. The results from the binomial logistic model indicated a significant effect of farm and therefore results were corrected for farm by introducing this factor in all the subsequent analyses. The SNP analysis on DNA from blood taken from the 553 animals present at the last sampling is summarized in Table 3. The global simultaneous association test presented a $P$ value $<0.001$, indicating that at least one marker or one combination of markers was associated with resistance/ susceptibility to paratuberculosis. The model with smallest BIC among all the possible models containing effects of farm and combinations of the five SNP markers was a model containing only farm and the marker SNP01 TLR2-1903 T/C, confirming the preliminary association found in the casecontrol study. The allelic distribution and disease association of the SNP01 TLR2-1903 T/C was confirmed $(P=0.01)$. Marginally significant effects of SNP03 TLR2-385 T/G $(P=0.06)$ and SNP05 TLR2-1828 C/T SNP $(P=0.06)$ were observed in models in which SNP01 was excluded. Combination models of farm, SNP01 and either or both SNP03 and SNP05 indicated that results were fully conditional on the observed association for SNP01 $(P<0.01)$ (Table 3). For the 
Table 3

Results of Toll-like receptor 2 (TLR2) single nucleotide polymorphism (SNP) typing of five different SNP in 553 cows from eight farms with endemic paratuberculosis and their association with disease.

\begin{tabular}{|c|c|c|c|c|c|c|c|c|c|c|c|}
\hline \multirow[t]{3}{*}{ TLR2 SNP ID } & \multirow[t]{3}{*}{ Position } & \multirow[t]{3}{*}{ Alleles } & \multicolumn{8}{|c|}{ Paratuberculosis status } & \multirow[t]{3}{*}{$P$-value ${ }^{a}$} \\
\hline & & & \multicolumn{4}{|c|}{ Test negative $(n=308)$} & \multicolumn{4}{|c|}{ Test positive $(n=245)$} & \\
\hline & & & HO1 & HET & $\mathrm{HO} 2$ & nd & HO1 & HET & $\mathrm{HO} 2$ & nd & \\
\hline SNP01 & 1903 & CC-CT-TT & 0 & 58 & 248 & 2 & 5 & 63 & 176 & 1 & 0.0096 \\
\hline SNP03 & 1828 & CC-CT-TT & 4 & 53 & 251 & 0 & 5 & 58 & 181 & 1 & 0.06 \\
\hline SNP05 & 385 & GG-GT-TT & 0 & 71 & 228 & 9 & 0 & 82 & 159 & 4 & 0.06 \\
\hline SNP06 & 651 & GG-GA-AA & 6 & 60 & 242 & 0 & 7 & 62 & 174 & 2 & n.s. \\
\hline SNP08 & 798 & GG-GA-AA & 6 & 60 & 242 & 0 & 7 & 59 & 177 & 2 & n.s. \\
\hline
\end{tabular}

The SNP, resulting alleles and respective counts in Mycobacterium avium subspecies paratuberculosis (Map)-test negative and Map-test positive animals are shown.

a Allele frequency differences were tested using binomial logistic models with the response variable given by a binary indicator of the paratuberculosis diagnosis; HO1 = first homozygous allele in alleles column; HET = heterozygous allele; HO2=second homozygous allele in alleles column; nd= not determined. A dominance model was used in which allele frequencies in which the SNP was present (sum of the first and second allele given) were compared to the allele frequencies without the SNP (last allele given). Disease associations, as indicated by allele frequency differences, were inferred using binomial logistic models containing the SNP and farm. The marginally significant associations for SNP03 and SNP05 $(P=0.06)$ were observed in models in which SNP01 was excluded. Combination models of farm, SNP01 and either or both SNP03 and SNP05 indicated that results were fully conditional on the observed association for SNP01 $(P<0.01)$. The level of statistical significance was set at $P<0.05$; n.s. $=$ not significant.

remaining two SNP no significant difference in allelic distribution between infected and non-infected animals was observed. In addition, none of all the possible combinations for the five SNP indicated a significant association for a single combination if SNP01 was not included in the model. For the TLR2-1903 T/C the Odds ratio (OR) was calculated by comparing the CT and CC genotypes to the TT genotype, similar to the dominance model used for calculating the association. That comparison resulted in an OR of 1.7 (95\% CI: $1.2,2.8)$ for animals to be Map infected given the presence of the TLR2-1903 T/C mutation (i.e., the CT and CC genotypes). No statistically significant interactions between farm and SNP markers were found.

\subsection{Functional consequences of SNP in bovine TLR2}

\subsubsection{Effect of SNP on intra-cellular signaling properties of TLR2}

The three genes that were selected to study functional effects of SNP had SNP in the extra-cellular LRR domain of the molecule only; the transmembrane and intracellular parts of these TLR2 molecules were identical. One TLR2 gene was cloned from a healthy animal (no disease association, identical to the reference sequence); additionally, two genes were selected from an animal with clinical signs of disease (TLR2K_S) and a healthy control animal (TLR2K_R), based on the TLR2-1903 T/C SNP types of these animals; CC and TT, respectively. All animals were also part of the case-control study.

The results of resequencing of the genes which were cloned into the pCMV-FLAG expression vector indicated that the genes of the diseased and the healthy cow were different with respect to TLR2 $1903 \mathrm{~T} / \mathrm{C}$ and three additional, previously identified positions leading to three non-synonymous point mutations as indicated in Fig. 1. It was also confirmed that all mutations were in extracellular parts of the TLR2 molecules; the transmembrane and intracellular signaling parts were identical (data not shown).

There was no detectable difference in expression of the three cloned genes in both stimulated and unstimulated
HEK293 cells, measured by flow cytometric detection of the N-terminal FLAG tag (data not shown).

Transfection of HEK293 cells with the TLR2 gene cloned from the healthy animal resulted in NFKB-driven luciferase activity that was up-regulated in a dose-dependent manner after stimulation of the cells with PAM3CSK4 and PAM2CSK4 (Fig. 2). In addition, responses were also observed upon stimulation of cells with Map and recombinant Map Hsp70 protein (data not shown). No activation was observed in the mock-transfected HEK293 cells.

Next, the effect of the susceptible (TLR2K_S) and the resistant (TLR2K_R) haplotypes on the functionality of TLR2, i.e., NFKB activity, was investigated. Stimulation with PAM3CSK4 resulted in NFKB activation with both haplotypes. The resistant haplotype showed significantly $(P<0.05)$ higher responses to PAM3CSK4, which was also observed upon stimulation with three tested Map concentrations (Fig. 3). The recombinant Map Hsp70 protein did induce NFKB activation, but although the resistant haplotype had consistently higher average responses no significant differences were observed (data not shown).

\subsubsection{Cytokine and TLR gene transcription in bovine monocytes}

Quantitative PCR was used to evaluate cytokine and TLR expression levels in monocytes derived from a group of Map-infected cattle negative for the TLR2-1903 T/C ('TT', $n=5$ ) and a group of Map-infected cattle positive for the TLR2-1903 T/C ('TC', $n=5$ ). In unstimulated cells (medium control) gene expression levels of TNF $\alpha$, IL1 $\beta$, IL10, IL12p40, TLR2 and TLR9 did not differ significantly. However, transcription of IL6 was downregulated sevenfold (95\% CI: 1.5, 32.3; $P=0.04$ ) in TLR2-1903 'TT' monocytes compared to TLR2-1903 'TC' monocytes. When monocytes were stimulated with apathogenic Map (moi 10:1), gene expression levels of TNF $\alpha$, IL1 $\beta$, IL6, IL10, IL12p40, TLR2 and TLR9 did not differ significantly. When monocytes were stimulated with Map (moi 10:1) transcription of genes was comparable with the exception of two genes. The IL1 $\beta$ transcription was upregulated 17-fold (95\% CI: 3.6, 5803.0; $P=0.03$ ) in TLR2-1903 'TT' monocytes 
TLR2K_S MPRALWTAWV WAVIILLTEGA SDQASSLSCD PTGVCDGHSR LNSIPSGLT

TLR2K_R MPRALWTAWV WAVIILLTEGA SDQASSLSCD PTGVCDGHSR LNSIPSGLT

TLR2K_S AGVKSLDLSN NDITYVGNRD LQRCVNLKTL RLGANEIHTV EEDSFFHLRN

TLR2K_R AGVKSLDLSN NEITYVGNRD LQRCVNLKTL RLGANEIHTV EEDSFFHLRN

t385g (D-E)

101

150

TLR2K_S LEYLDLSYNR LSNLSSSWFR SLYVLKFLNL LGNLYKTLGE TSLFSHLPNL

TLR2K_R LEYLDLSYNR LSNLSSSWFR SLYVLKFLNL LGNLYKTLGE TSLFSHLPNL

g651a (R-Q)

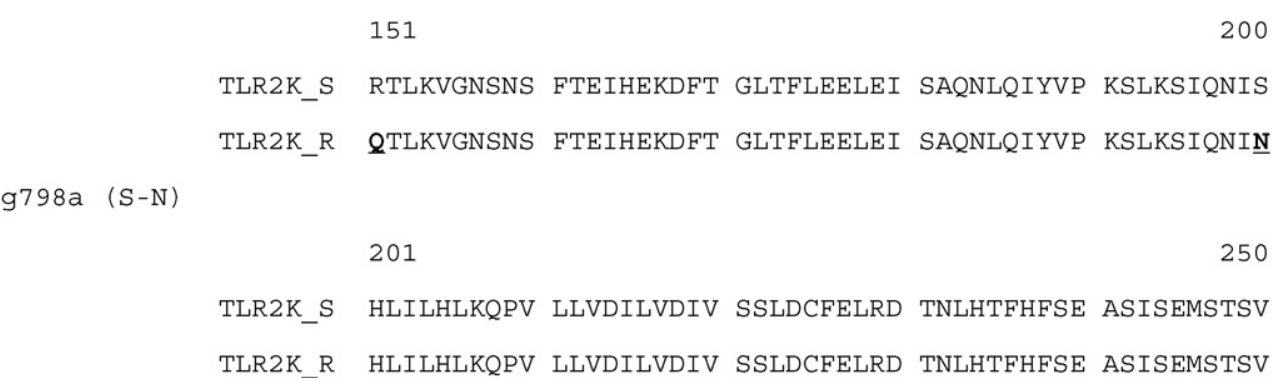

Fig. 1. Differences at protein level between the cloned Toll-like receptor 2 (TLR2) molecules from cows with clinical signs of paratuberculosis (TLR2K_S) and resistance to paratuberculosis infection (TLR2K_R). The first 250 amino acids of the TLR2 protein (773 amino acids) are shown. The signal peptide sequence is underlined, and from amino acid 12 onwards the sequence was cloned into the pCMV-FLAG expression plasmid and confirmed by sequencing for each cloned molecule. Differences at amino acid level between resistant and susceptible genetic variants are indicated by bold-underlined amino acids in the resistant TLR2. The corresponding single nucleotide polymorphism (SNP) is indicated on the left. In addition, these two molecules differed for SNP TLR21903 T/C: TLR2K_S had genotype 'CC' and TLR2K_R had genotype 'TT'. The TLR2-1903 T/C SNP does not lead to a change in amino acid sequence.

compared to TLR2-1903 'TC' monocytes. The IL12p40 transcription was upregulated 8.9-fold (95\% CI: 1.9, 32.0; $P=0.04)$ in TLR2-1903 'TT' monocytes compared to TLR21903 'TC' monocytes.

\subsubsection{Antigen specific $T$ cell and $B$ cell responses}

The proliferation assay was used to evaluate $\mathrm{T}$ cell proliferation from paratuberculosis infected cattle to mitogen and PPD antigen. Mononuclear lymphocytes were derived from a group of Map-infected cattle negative for the TLR2-1903 T/C ('TT', $n=35$ ) and a group of Mapinfected cattle positive for the TLR2-1903 T/C ('CT', $n=15$ ). The proliferation in response to aspecific mitogen stimulation was not significantly different when comparing both groups. In contrast, TLR2-1903 'CT' animals showed a significantly $(P=0.03)$ lower proliferation to

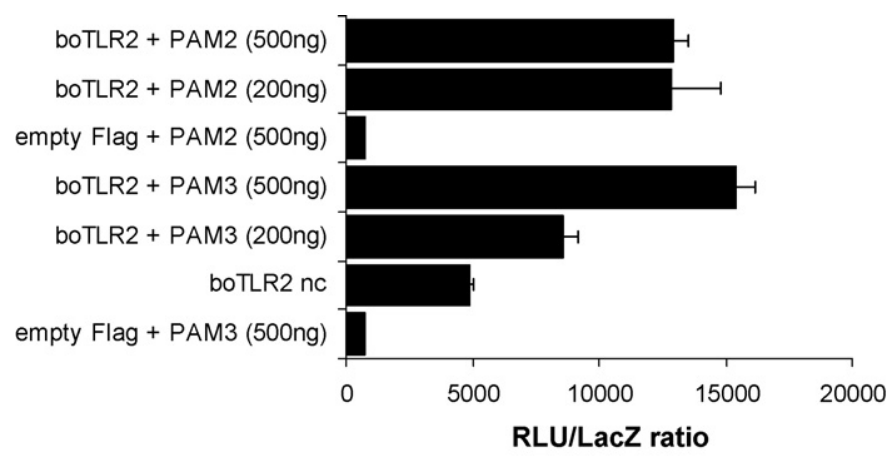

Fig. 2. The functionality of bovine Toll-like receptor 2 (TLR2) molecules in HEK293 cells transfected with the pFLAG-CMV-1-boTLR2 (boTLR2) or empty pFLAG vector (empty Flag), luciferase and LacZ reporter vectors is shown. Transfected cells were stimulated with ligands PAM2CSK4 (PAM2) or PAM3CSK4 (PAM3) at indicated concentrations, or not stimulated (bo TLR2 nc). A representative example of three independent experiments is shown. Luciferase activity was measured as relative light units (RLU) and corrected for transfection efficacy based on the LacZ reporter gene activity (RLU/LacZ ratio).

Please cite this article in press as: Koets, A., et al., Susceptibility to paratuberculosis infection in cattle is associated with single nucleotide polymorphisms in Toll-like receptor 2 which modulate immune responses against Mycobacterium avium subspecies paratuberculosis. Prev. Vet. Med. (2009), doi:10.1016/j.prevetmed.2009.11.008 


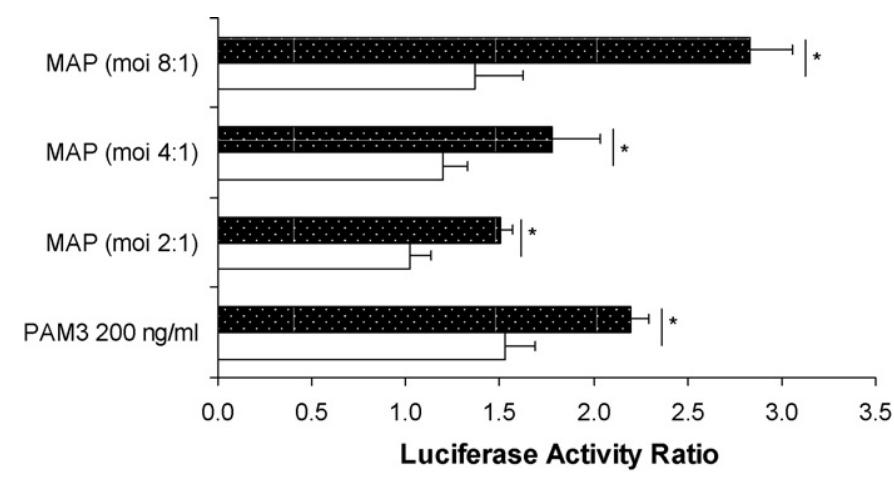

Fig. 3. The functional effects of susceptible (light bars) and resistant (dark bars) Toll-like receptor 2 haplotypes are shown when stimulated with indicated concentrations of the ligands PAM3CSK4 (PAM3), Mycobacterium avium subspecies paratuberculosis (Map) [at three multiplicities of infection (moi) indicating the number of Map cfu per cell]. The luciferase activity was measured in relative light units, corrected for transfection efficiency (LacZ) and related to unstimulated controls; the ratio is expressed as the luciferase activity ratio. Results of three independent experiments were combined. Statistically significant differences $(P<0.05)$ between sensitive and resistant haplotypes using Students $t$-test are indicated $\left({ }^{*}\right)$.

mycobacterial PPD antigen (average stimulation index (S.I.) $4.2 \pm 1.9 \mathrm{SD}$ ) compared to TLR2-1903 'TT' animals (average S.I. $7.6 \pm 8.3 \mathrm{SD}$ ).

Map-specific antibodies in serum of TLR2-1903 'CT' animals showed an average sample to positive $(\mathrm{S} / \mathrm{P})$ ratio of $234 \pm 54$ SD, and an average S/P ratio of $241 \pm 49$ SD in TLR21903 'TT' animals as measured with the Pourquier absorbed ELISA, and were not significantly different between the two groups.

\section{Discussion}

The results of the current study indicate that polymorphisms in the TLR2 gene are likely to be involved in susceptibility to bovine paratuberculosis. In the casecontrol study the cases were cows with positive fecal culture that also showed symptoms of clinical paratuberculosis and as such an extreme phenotype of the disease. The case-control study indicated an OR of six for the genetic risk factor SNP01 TLR2-1903 T/C. The post hoc power calculations on the matched case-control study indicated that if the genetic risk factor had a Mendelian distribution, which was a used as $\mathrm{H}(0)$ due to lack of data, the power of the study would not be sufficient to detect an OR of two and the case-control could only have served as the basis for TLR2 SNP discovery in 24 cows. However, as the genetic risk factor was far less common than predicted by the $\mathrm{H}(0)$, a significant difference was found. In the farmlevel observational field study no records were available regarding the presence or absence of symptoms and classification of animals was exclusively based on combination of ELISA and fecal culture results. No power calculations were performed prior to experiment as it was decided to study the entire available cohort. In retrospect the genetic risk factor, as defined by the SNP01 TLR2-1903 T/C was less frequent compared to the $\mathrm{H}(0)$ but with $23 \%$ not rare. Post hoc power calculations indicated that to detect a OR of 2.0 , with $95 \%$ confidence and $80 \%$ power, assuming 40\% non-exposed diseased animals, 20 animals were required in each group, indicating sufficient power for our studies. In the observational field study using a total of 553 cows, significant differences in allelic distribution of genetic polymorphisms in TLR2, in particular SNP01 TLR2-1903 T/C, were found that confirmed results of the case-control study.

The ratio non-synonymous:synonymous SNP indicates whether non-synonymous polymorphisms have caused a negative selection (ratio $<1$ ), a positive selection (ratio $>1$ ) or whether they are neutral (ratio $=1$ ) (Yang et al., 2000). The ratio of 2.2:1, which we found with respect to TLR2 SNP indicates a positive selection for nonsynonymous polymorphisms in bovine TLR2. Average frequencies of one SNP per 689 bp regarding all SNP, and one non-synonymous SNP per $1009 \mathrm{bp}$ were found. Previous studies on other genes in cattle have reported higher SNP frequencies with an average of one SNP per 143 bp (Heaton et al., 2001) and one SNP per 90 bp (White et al., 2003). However, our data are consistent with SNP frequencies described in humans, where frequencies range from one SNP per 657 bp (Wang et al., 1998), to one SNP per 1001 bp (Lai et al., 1998), in the total human genome. The putative LRR domain of bovine TLR2 has the highest average SNP frequency. These data are in line with studies in bovine, human and mouse TLR4 (Smirnova et al., 2000, 2001; White et al., 2003). In addition, the small number of non-synonymous SNP in the transmembrane and TIR domains confirms data about those conserved domains.

Previous studies in humans have shown that mutations in TLR2 confer susceptibility to mycobacterial infections. Two mutations in TLR2, an Arg753Gln polymorphism and an Arg677Trp polymorphism, have been associated with susceptibility to disease (Lorenz et al., 2000; Kang and Chae, 2001). The Arg753Gln polymorphism has been associated with a negative influence on TLR2 signalling, increasing the risk of mycobacterial infections (Mycobacterium leprae and Mycobacterium tuberculosis) as well as infections with Gram-positive (Staphylococcus aureus) and Gram-negative (Borrelia burgdorferi) bacteria (Lorenz et al., 2000; Ogus et al., 2004). The Arg677Trp mutation is probably involved in determining the type of leprosy, because it was significantly more prevalent in patients with lepromatous leprosy than with tuberculoid leprosy (Kang and Chae, 2001; Ben-Ali et al., 2004). Both mutations are located at the C-terminus of the TLR2, in the TIR 
domain, and could affect the intracellular signalling function of the TLR. Bochud et al. (2003) have shown that the Arg677Trp mutation prohibits the Toll-pathway and production of NFKB. Although the mutations found in the current study are not in the intra-cellular C-terminus of TLR2, the results indicate that the mutations in the extracellular LRR also influence TLR2 signalling and production of NFKB.

A recent study of Pinedo and colleagues revealed SNP in the CARD15 gene in cattle which were associated with susceptibility to paratuberculosis (Pinedo et al., 2008). The associations observed in that study are similar to the associations observed in the TLR2 SNP estimated in the current study. The OR of the CARD15 C733R was 3.35 in the study by Pinedo et al. (2008) and approximately twice as high as the OR for the TLR2-1903 T/C. The SNP TLR2-1903 T/C and TLR2-1828 T/C which were most significantly associated with infection status are both synonymous and do not lead to amino acid changes.

Data in the current study on the function of TLR2 confirm that PAM3CSK4 is recognized by bovine TLR2 (Aliprantis et al., 1999) and that PAM2CSK4 is able to signal via TLR2 without TLR6 (Buwitt-Beckmann et al., 2005). Stimulation with Map, of which some cell wall components are ligands for TLR2, resulted in a lower response compared to the response at PAM3CSK4 and PAM2CSK4. This difference may be due to the concentrations used as PAM3CSK4 and PAM2CSK4 are purified, synthetic ligands in contrast to Map. Comparing the responses of cells transfected with TLR2, derived from cows with SNP associated with a TLR2-1903 'CT' versus TLR2-1903 'TT', the latter showed the highest activity after stimulation with PAM3CSK4 and especially after stimulation with Map. The other three SNP in TLR2 represented in this TLR2-1903 'CT' versus TLR2-1903 'TT' comparison are non-synonymous and these SNP lead to amino acid changes with functional effects. These SNP are in the extracellular LRR part of TLR2 and lead to attenuation of NFKB signalling. The mutation CARD15 C733R, identified in the study of Pinedo et al. (2008), in this intracellular bacterial receptor may lead to attenuated NFKB signalling, as has been reviewed by Strober et al. (2006), although this has not been studied in cattle.

The functional studies support the hypothesis that an attenuated TLR2 function is a risk factor for developing clinical paratuberculosis. The results from the cytokine transcription analysis in monocytes indicated that monocytes of TLR2-1903 'TT' genotype animals are more prone to the production of pro-inflammatory Th1 driving cytokines IL1 $\beta$ and IL12p40 when stimulated with Map. These differences were not attributable to differences in expression of TLR2 as expression levels were similar between the different groups, and do not seem to be caused by differential transcriptional regulation of the TLR2 gene in the cattle groups studied. Recent studies by Weiss and colleagues (Weiss et al., 2007) suggest an important role for TLR2 in regulating the intracellular fate of Map. The macrophage data suggest an inherent increased transcription of immunosuppressive IL6 transcription by TLR2-1903 'CT' (susceptibility associated genotype) monocytes. Similar to observations in the study by Weiss et al. (2007) no differential transcription of IL10 was observed. Our finding of relative upregulation of IL6 is in agreement with studies in TLR2-/- mice suggesting a role of TLR2 in immunosuppression during mycobacterial infection via upregulation of IL6 (Jang et al., 2004), which may also play a role in cattle.

In addition, the adaptive immune response in TLR21903 'TT' animals is also more prone to a Th1-like phenotype considering higher proliferative $\mathrm{T}$ cell responses in these animals compared to TLR2-1903 'CT' animals. Antibody levels appear similar in the animals studied arguing against an overall switch of Th1 to Th2 adaptive responses.

In conclusion, in cows with a susceptible TLR2 haplotype, the clinical phase of paratuberculosis may start at a younger age, or may be more severe, as a consequence of inadequate innate and subsequent cellmediated immune responses. However, more research into the functional effects of SNP and the downstream effects on protective Th1-type responses is needed and, as suggested by other studies, combined polygenic effects are likely involved in the pathogenesis. Particularly a combined study of the CARD15 and TLR2 polymorphisms should be considered since they clearly point to functional differences in macrophages in which mycobacteria manage to survive bacteriocidal mechanisms. Ultimately, the TLR2 and additional SNP typing may be useful in marker-assisted breeding strategies, screening for risks for disease and improving the genetic resistance of cattle to paratuberculosis, although care should be taken not increase susceptibility to other diseases by one particular selection focus. Nonetheless, breeding of more resistant animals can be used as an additional method to control paratuberculosis in cattle populations besides management, test and cull and vaccination strategies.

\section{Conflict of interest}

A.F. is a full time employee of the research and development department of CRV BV (an international enterprise in the field of cattle improvement). He was a technical consultant for the genetics part of the work. Neither CRV BV nor the Technology Foundation was involved in the study design, data collection, analysis or interpretation of data, writing of the manuscript or decision to submit the manuscript for publication. The remaining authors declare no conflict of interest.

\section{Acknowledgement}

This study was supported by a grant from the Technology Foundation (STW) of the Dutch Research Council (NWO), grant number STW-UDG5589.

\section{References}

Aliprantis, A.O., Yang, R.B., Mark, M.R., Suggett, S., Devaux, B., Radolf, J.D. Klimpel, G.R., Godowski, P., Zychlinsky, A., 1999. Cell activation and apoptosis by bacterial lipoproteins through toll-like receptor-2. Science $285,736-739$.

Alzuherri, H.M., Little, D., Clarke, C.J., 1997. Altered intestinal macrophage phenotype in ovine paratuberculosis. Res. Vet. Sci. 63, 139-143. 
Ben-Ali, M., Barbouche, M.R., Bousnina, S., Chabbou, A., Dellagi, K., 2004. Toll-like receptor 2 Arg677Trp polymorphism is associated with susceptibility to tuberculosis in Tunisian patients. Clin. Diagn. Lab. Immunol. 11, 625-626.

Burnham, K.P., Anderson, D.R., 2002. Model Selection and Multimodel Inference: A Practical-Theoretic Approach, 2nd ed. Springer-Verlag, ISBN: 0-387-95364-7.

Bochud, P.Y., Hawn, T.R., Aderem, A., 2003. Cutting edge: a Toll-like receptor 2 polymorphism that is associated with lepromatous leprosy is unable to mediate mycobacterial signaling. J. Immunol. 170, 3451-3454.

Buwitt-Beckmann, U., Heine, H., Wiesmuller, K.H., Jung, G., Brock, R., Akira, S., Ulmer, A.J., 2005. Toll-like receptor 6-independent signaling by diacylated lipopeptides. Eur. J. Immunol. 35, 282-289.

Casanova, J.L., Abel, L., 2002. Genetic dissection of immunity to mycobacteria: the human model. Annu. Rev. Immunol. 20, 581-620.

Coussens, P.M., 2004. Model for immune responses to Mycobacterium avium subspecies paratuberculosis in cattle. Infect. Immun. 72, 30893096.

Estonba, A., Iriondo, M., Oyanguren, O., Garrido, J.M., Geijo, M.V., Sevilla, I., Juste, R.A., 2005. Bovine 3'-UTR (GT11) variant of the NRAMP1 gene under-represented among paratuberculosis seropositive cattle. In: Proceedings of the 8th International Colloquium on Paratuberculosis.

Gonda, M.G., Chang, Y.M., Shook, G.E., Collins, M.T., Kirkpatrick, B.W. 2006. Genetic variation of Mycobacterium avium ssp. paratuberculosis infection in US Holsteins. J. Dairy Sci. 89, 1804-1812.

Gonda, M.G., Kirkpatrick, B.W., Shook, G.E., Collins, M.T., 2007. Identification of a QTL on BTA20 affecting susceptibility to Mycobacterium avium ssp. paratuberculosis infection in US Holsteins. Anim. Genet. $38,389-396$.

Greenstein, R.J., 2003. Is Crohn's disease caused by a mycobacterium? Comparisons with leprosy, tuberculosis, and Johne's disease. Lancet Infect. Dis. 3, 507-514.

Haughton, D.M.A., 1988. On the choice of a model to fit data from an exponential family. Ann. Stat. 16, 342-355.

Heaton, M.P., Grosse, W.M., Kappes, S.M., Keele, J.W., Chitko-McKown, C.G., Cundiff, L.V., Braun, A., Little, D.P., Laegreid, W.W., 2001. Estimation of DNA sequence diversity in bovine cytokine genes. Mamm Genome 12, 32-37.

Hostetter, J., Steadham, E., Haynes, J., Bailey, T., Cheville, N., 2003. Phagosomal maturation and intracellular survival of Mycobacterium avium subspecies paratuberculosis in J774 cells. Comparat. Immunol., Microbiol. Infect. Dis. 26, 269-283.

Jang, S., Uematsu, S., Akira, S., Salgame, P., 2004. IL-6 and IL-10 induction from dendritic cells in response to Mycobacterium tuberculosis is predominantly dependent on TLR2-mediated recognition. J. Immunol. 173, 3392-3397.

Jorgensen, J.B., 1982. An improved medium for culture of Mycobacterium paratuberculosis from bovine faeces. Acta Vet. Scand. 23, 325-335.

Kalinski, P., Hilkens, C.M., Wierenga, E.A., Kapsenberg, M.L., 1999. T-cell priming by type- 1 and type- 2 polarized dendritic cells: the concept of a third signal. Immunol. Today 20, 561-567.

Kang, T.J., Chae, G.T., 2001. Detection of Toll-like receptor 2 (TLR2) mutation in the lepromatous leprosy patients. FEMS Immunol. Med. Microbiol. 31, 53-58.

Keestra, A.M., de Zoete, M.R., van Aubel, R.A., van Putten, J.P., 2007. The central leucine-rich repeat region of chicken TLR16 dictates unique ligand specificity and species-specific interaction with TLR2. J. Immunol. 178, 7110-7119.

Koets, A., Rutten, V., Hoek, A., van Mil, F., Muller, K., Bakker, D., Gruys, E., van Eden, W., 2002. Progressive bovine paratuberculosis is associated with local loss of CD4(+) T cells, increased frequency of gamma delta T cells, and related changes in T-cell function. Infect. Immun. 70, 3856-3864.

Koets, A.P., Adugna, G., Janss, L.L., van Weering, H.J., Kalis, C.H., Wentink, G.H., Rutten, V.P., Schukken, Y.H., 2000. Genetic variation of susceptibility to Mycobacterium avium subsp. paratuberculosis infection in dairy cattle. J. Dairy Sci. 83, 2702-2708.

Koets, A.P., Rutten, V.P., Hoek, A., Bakker, D., van Zijderveld, F., Muller, K.E., van Eden, W., 1999. Heat-shock protein-specific T-cell responses in various stages of bovine paratuberculosis. Vet. Immunol. Immunopathol. 70, 105-115.

Kuehnel, M.P., Goethe, R., Habermann, A., Mueller, E., Rohde, M., Griffiths, G., Valentin-Weigand, P., 2001. Characterization of the intracellular survival of Mycobacterium avium ssp. paratuberculosis: phagosomal $\mathrm{pH}$ and fusogenicity in $\mathrm{J774}$ macrophages compared with other mycobacteria. Cell Microbiol. 3, 551-566.

Labouriau, R., Sørensen, P., Juul-Madsen, H.R., 2008. An efficient strategy to characterize alleles and complex haplotypes using DNA-markers. arXiv:0804.1690v1 [Stat.AP].
Langelaar, M.F., Weber, C.N., Overdijk, M.B., Muller, K.E., Koets, A.P., Rutten, V.P., 2005. Cytokine gene expression profiles of bovine dendritic cells after interaction with Mycobacterium avium ssp. paratuberculosis (M.a.p.), Escherichia coli (E. coli) or recombinant M.a.p. heat shock protein 70. Vet. Immunol. Immunopathol. 107, 153-161.

Lai, E., Riley, J., Purvis, I., Roses, A., 1998. A 4-Mb high density single nucleotide polymorphism based map around human APOE. Genomics 54, 31-38.

Lorenz, E., Mira, J.P., Cornish, K.L., Arbour, N.C., Schwartz, D.A., 2000. A novel polymorphism in the Toll-like receptor 2 gene and its potential association with staphylococcal infection. Infect. Immun. 68, 6398-6401.

Ogus, A.C., Yoldas, B., Ozdemir, T., Uguz, A., Olcen, S., Keser, I., Coskun, M., Cilli, A., Yegin, O., 2004. The Arg753GLn polymorphism of the human Toll-like receptor 2 gene in tuberculosis disease. Eur. Respir. J. 23, 219-223.

Pfaffl, M.W., Horgan, G.W., Dempfle, L., 2002. Relative expression software tool (REST) for group-wise comparison and statistical analysis of relative expression results in real-time PCR. Nucleic Acids Res. 30, e36.

Pinedo, P.J., Buergelt, C.D., Donovan, G.A., Melendez, P., Morel, L., Wu, R., Langaee, T.Y., Rae, D.O., 2008. Association between CARD15/NOD2 gene polymorphisms and paratuberculosis infection in cattle. Vet. Microbiol.

Quesniaux, V., Fremond, C., Jacobs, M., Parida, S., Nicolle, D., Yeremeev, V., Bihl, F., Erard, F., Botha, T., Drennan, M., Soler, M.N., Le Bert, M., Schnyder, B., Ryffel, B., 2004. Toll-like receptor pathways in theimmune responses to mycobacteria. Microbes Infect. 6, 946-959.

R Development Core Team, 2008. R: A Language and Environment for Statistical computing. R Foundation for Statistical Computing, Vienna, Austria. ISBN 3-900051-07-0, http://www.R-project.org.

Rozen, S., Skaletsky, H., 2000. Primer3 on the WWW for general users and for biologist programmers. In: Krawetz, S., Misener, S. (Eds.), Bioinformatics Methods and Protocols: Methods in Molecular Biology. Humana Press, Totowa, NJ, pp. 365-386.

Smirnova, I., Hamblin, M.T. McBride, C., Beutler, B., Di Rienzo, A., 2001. Excess of rare amino acid polymorphisms in the Toll-like receptor 4 in humans. Genetics 158, 1657-1664.

Smirnova, I., Poltorak, A., Chan, E.K., McBride, C., Beutler, B., 2000. Phylogenetic variation and polymorphism at the Toll-like receptor 4 locus (TLR4). Genome Biol. 1 RESEARCH002.

Strober, W., Murray, P.J., Kitani, A., Watanabe, T., 2006. Signalling pathways and molecular interactions of NOD1 and NOD2. Nat. Rev. Immunol. 6, 9-20.

Wang, D.G., Fan, J.B., Siao, C.J., Berno, A., Young, P., Sapolsky, R., Ghandour, G., Perkins, N., Winchester, E., Spencer, J., Kruglyak, L., Stein, L., Hsie, L., Topaloglou, T., Hubbell, E., Robinson, E., Mittmann, M., Morris, M.S. Shen, N., Kilburn, D., Rioux, J., Nusbaum, C., Rozen, S., Hudson, T.J., Lipshutz, R., Chee, M., Lander, E.S., 1998. Large-scale identification, mapping, and genotyping of single-nucleotide polymorphisms in the human genome. Science 280, 1077-1082.

Weckx, S., Del-Favero, J., Rademakers, R., Claes, L., Cruts, M., De Jonghe, P., Van Broeckhoven, C., De Rijk, P., 2005. novoSNP, a novel computational tool for sequence variation discovery. Genome Res. 15, 436-442.

Weiss, D.J., Evanson, O.A., de Souza, C., Abrahamsen, M.S., 2005. A critical role of interleukin-10 in the response of bovine macrophages to infection by Mycobacterium avium subsp. paratuberculosis. Am. J. Vet. Res. 66, 721-726.

Weiss, D.J., Evanson, O.A., Deng, M., Abrahamsen, M.S., 2004. Gene expression and antimicrobial activity of bovine macrophages in response to Mycobacterium avium subsp. paratuberculosis. Vet. Pathol. 41, 326-337.

Weiss, D.J., Souza, C.D., Evanson, O.A., Sanders, M., Rutherford, M., 2007. Bovine monocyte TLR2 receptors differentially regulate the intracellular fate of Mycobacterium avium subsp. paratuberculosis and Mycobacterium avium subsp. avium. J. Leukoc. Biol.

Werling, D., Hope, J.C., Howard, C.J., Jungi, T.W., 2004. Differential production of cytokines, reactive oxygen and nitrogen by bovine macrophages and dendritic cells stimulated with Toll-like receptor agonists. Immunology 111, 41-52.

Werling, D., Piercy, J., Coffey, T.J., 2006. Expression of Toll-like receptors (TLR) by bovine antigen-presenting cells: Potential role in pathogen discrimination? Vet. Immunol. Immunopathol., Innate Immun. Vacc. $112,2-11$

White, S.N., Taylor, K.H., Abbey, C.A., Gill, C.A., Womack, J.E., 2003. Haplotype variation in bovine Toll-like receptor 4 and computational prediction of a positively selected ligand-binding domain. Proc. Natl. Acad. Sci. U.S.A. 100, 10364-10369.

Yang, Z., Nielsen, R., Goldman, N., Pedersen, A.M., 2000. Codon-substitution models for heterogeneous selection pressure at amino acid sites. Genetics 155, 431-449. 